\title{
L'oral imité dans la littérature, un objet didactique réflexif en classe de langues étrangères voisines
}

\author{
Typhaine Manzato ${ }^{1, \mathrm{a}^{*}}$ \\ ${ }^{1}$ Université de Bordeaux, Laboratoire LACES EA 7437, 33076 Bordeaux Cédex, France - Université \\ d'Aix-Marseille, Laboratoire LPL UMR 7309, 13100 Aix-en-Provence, France
}

\begin{abstract}
Résumé. Comment pourrait-on utiliser l'imitation de la langue parlée dans la littérature en vue d'un travail sur la compréhension des usages oraux de la langue étrangère en classe ? Cette proposition de communication peut être résumée par cette question et notre article sera guidé par la réponse que nous y apporterons. En particulier, nous montrerons comment a été mise à profit l'imitation de certains traits de la langue parlée dans des dialogues de romans dans le cadre de l'expérimentation de notre travail de thèse. Notre postulat est que la rencontre avec certains traits de la langue parlée par leur représentation littéraire à l'écrit - avec l'apport de leur double présentation à l'aide d'extraits de corpus oraux - pourrait aider à l'identification, en plus de permettre une réflexion, de ces phénomènes, de manière à pouvoir les reconnaitre ensuite à l'oral. Notre expérimentation consiste à observer l'apport de la présentation de ces faits dans une double modalité écrite et orale mais aussi dans une modalité plurilingue, avec la présence d'un même fait - ou même type de fait - en deux autres langues romanes, en complément des extraits en langue cible.
\end{abstract}

\begin{abstract}
Spoken language imitation in literature, a reflexive teaching material in class. How could one use the imitation of the spoken language in literature in order to understand the oral uses of the foreign language ? This communication can be summarized by this question and our article will be guided by the answer we give. More specifically, we will show how the imitation of certain features of the spoken language in novels' dialogues has been used in class as part of the experimentation of our doctoral research. Our postulate is that the presentation of a selection of certain features of the spoken language by their imitation in literature - with the contribution of their double presentation using extracts from spoken corpora - could help with the identification, in addition to allow a reflection, of these phenomena, in order to be able to recognize them in listening comprehension. Our experiment aims to observe the contribution of the presentation of these phenomenon in a double modality - written and oral - but also in a plurilingual modality, with the presentation of the same linguistic phenomenon - or type of phenomenon - in two other Romance languages, in addition to the extracts in target language.
\end{abstract}

\section{Introduction}

*typhaine.manzato@u-bordeaux.fr 
L'imitation de la langue parlée dans la littérature est un procédé intéressant pour plusieurs raisons: non seulement dans l'analyse qui peut en être faite concernant les buts des écrivain-e-s et leurs raisons d'imiter la langue parlée à l'écrit, mais aussi quant aux caractéristiques linguistiques de la langue imitée.

Dans le cadre de notre recherche de doctorat et suite aux postulats de plusieurs travaux réalisés sur cet objet (Weber, 2013 ; Calvi, 1996) nous ajoutons un troisième point qui est l'utilisation pédagogique qui peut être faite de ces imitations en classe, dans le but de proposer une réflexion sur les usages oraux de la langue étrangère.

À cela s'ajoute la visée originale de notre travail qui est celle de donner une perspective intercompréhensive en présentant les phénomènes linguistiques sélectionnés en plusieurs langues romanes, dans le cadre d'une expérimentation en classe de langues étrangères voisines - le français et l'italien - pour un public de lycéen-n-e-s entre la France et l'Italie.

Cet article s'ouvrira sur une discussion des principales caractéristiques de ce que Luzzati et Luzzati (1987) nomment le « style oralisé ». Une observation de la langue imitée par les auteur-e-s sera l'objet de la seconde partie, en confrontant deux extraits de dialogues tirés de deux romans d'époques différentes. La troisième partie concernera l'exploitation didactique des imitations de l'oral en littérature, en tenant compte des conclusions du chapitre précédent, et en proposant une réflexion entre l'articulation entre l'oral et l'écrit et la perspective intercompréhensive de notre étude. Pour terminer, la mise en place de l'utilisation concrète de ce matériel en classe sera présentée, avec les premières remarques à la suite de notre terrain en vue de l'analyse des données.

\section{L'imitation de la langue parlée en littérature}

\subsection{Un artefact littéraire qui s'efforce de reproduire la langue orale}

L'imitation de l'oralité dans la littérature est un procédé ancien mais qui a depuis le $19^{\text {ème }}$ siècle connu un "engouement sans précédent» (Durrer, 1996: 63), confirmé dans la littérature scientifique par les principales analyses qui se focalisent sur un corpus de romans allant du $19^{\text {ème }}$ au $21^{\text {ème }}$, avec une importante production des écrivain-e-s du $20^{\text {ème }}$ siècle.

Luzzati et Luzzati (1987: 15) désignent l'imitation de l'oral en littérature comme " style oralisé », et semblent être les premiers à le définir par cette appellation; celle-ci sera reprise par Durrer (2005: 25), qui parlera également d' "artefact», en tant que « sélection de traits qui connotent l'oralité » effectuée par les écrivain-e-s.

Si le «style oralisé » s'appuie sur l'idée d'oralité qui se dégage de l'écriture des écrivain-e-s, le terme d' " artefact» est plus précis et comporte une mise en garde pour rappeler que la représentation de la langue parlée dans un dialogue de roman et la langue parlée d'une conversation authentique ne sauraient être identiques (Durrer, 2005 : 39) ; nous reviendrons sur cette idée dans la deuxième partie.

Ce style va donc consister à sélectionner un certain nombre de caractéristiques « réelles ou imaginaires » dans l'intention de rendre l'oral à l'écrit (Durer, $2005: 39$ ), des domaines de la langue allant de la syntaxe à la phonétique en passant par le lexique, chaque champ ayant ses particularités et étant utilisé de façon différente selon les auteur-e-s.

La prononciation imitée est qualifiée comme un "trait particulièrement frappant », donnant «un indéniable effet d'oralité » (Durrer, $2005: 25)$, rendue à l'écrit grâce à «quelques ajustements orthographiques» (Rouayrenc, $2010: 243$ ). Dans les phénomènes ayant attrait à la prononciation, on retrouve les élisions de voyelles et le marquage de l'effacement du «e » caduc (Rouayrenc, 2010 : 243-244).

Les phénomènes syntaxiques imités sont plus subtils mais restent des marques « extrêmement importantes » dans l'idée de la syntaxe comme architecture de la langue 
(Durrer, $2005: 30$ ) ; les dislocations sont avec les constructions clivées les marques syntaxiques les plus souvent retrouvées dans les imitations (Durrer, 2005 : 33).

Enfin, le lexique est la composante qui «connote le plus fortement l'oral» et fait souvent figure de domaine privilégié voire seul domaine pour « soutenir l'effet de langue orale, familière le plus souvent, argotique parfois » (Durrer, 2005 : 34). Rouayrenc (2010: 241-242) distingue pour le lexique la représentation d'un oral « qui se veut courant » plutôt orienté vers une langue familière de l'oral «marqué » faisant apparaitre des marques d'argot.

L'utilisation du lexique est néanmoins discutée et le recours à un lexique familier d'auteurs comme Queneau et Céline fait dire à Luzzati et Luzzati (1987: 21) que le vocabulaire familier voire argotique ne suffit pas à représenter l'oral à l'écrit, et de ce fait l' « oralité » du style de ces auteurs est « sujette à caution ».

De même, la suppression du «e » caduc imitée par les écrivain-e-s est la marque la plus intéressante à l'écrit selon Vigneau-Rouayrenc (1991: 33), dans le «paradoxe» qu'elle représente en pouvant connoter au mieux une réplique comme parler populaire alors qu'elle peut en fait imiter une prononciation tout à fait correcte.

\subsection{L'imitation sous une loupe européenne}

Le style oralisé ne semble pas connaître de frontières, comme le prouve l'intérêt de la littérature européenne pour cet objet: que ce soit en Italie, en Espagne ou au Portugal, nombreux sont les écrivain-e-s européen-n-e-s à avoir sélectionné les traits d'oralité de leurs langues respectives pour les intégrer à leurs récits.

Notre recherche concernant l'enseignement du français et de l'italien, nous nous sommes concentrées en particulier sur les écrivain-e-s de ces deux pays, et dans une moindre mesure sur les écrivain-e-s espagnol-e-s et portugais-e-s pour notre corpus.

Pour l'Italie, nous nous appuyons sur les réflexions de Calaresu (2005: 113) qui analyse des extraits d'un corpus d'auteur-e-s du $20^{\text {ème }}$ au tout début du $21^{\text {ème }}$ siècle ; selon l'autrice, la simulation de la langue italienne parlée la plus 'réelle' est à trouver chez des auteur-e-s contemporains comme Paolo Nori et Rosanna Campo, mais on retrouve cette problématique également chez les écrivain-e-s néoréalistes ou inspirés du courant néoréaliste à l'instar de Carlo Cassola ${ }^{\mathrm{i}}$.

En Espagne, les études sur l'imitation de la langue parlée en littérature concernent de façon importante les romans d'écrivain-e-s espagnols de l'après-guerre, dont le travail de López Serena (2007). L'autrice aborde la question de la syntaxe de la langue parlée avec des extraits de deux romans écrits pendant cette période par Rafael Sánchez Ferlosio et Carmen Martin Gaite, en comparant les textes à l'analyse syntaxique d'extraits oraux authentiques tirés d'un corpus de langue espagnole.

Duarte (2012 : 460) prend la même voie dans son étude sur le Portugal en comparant des données orales avec un corpus d'auteur-e-s contemporain-e-s tels José Saramago, José Cardoso Pires et Olga Gonçalves, ou plus anciens comme Miguel Torga, et Eça de Queirós, en concluant que l'imitation de l'oral en littérature reste une « construction fictive écrite ».

Il semblerait qu'il n'existe pas pour le moment d'études rassemblant des analyses d'imitations romanesques de l'oral en plusieurs langues, même si des événements européens - voire mondiaux - ont souvent fait figure d'arrière-plan dans les récits, comme les deux guerres mondiales et les périodes intermédiaires aux conflits.

En France, on retrouve la période du « roman parlant » théorisée par Meizoz (2001) qui correspond aux années 1919-1939, alors que les néoréalistes italiens apparaissent à partir de la fin de la seconde guerre mondiale, et l'après seconde guerre mondiale semble être une période propice aux imitations de la langue parlée dans la littérature en Espagne. 


\subsection{Une imitation revendicatrice et esthétique}

L'imitation de la langue parlée en littérature est un procédé poursuivant plusieurs vocations en lien avec le contexte de l'œuvre produite et les souhaits de son auteur-e.

En particulier, elle constitue une revendication à plusieurs niveaux : dans un premier temps, elle est le renouveau d'une esthétique littéraire que les écrivain-e-s cherchent à bousculer en lien avec l'intégration de l'oral en littérature venant questionner les normes littéraires, dans le cadre d'une réflexion plus générale sur la figure du peuple dans le roman.

Ces revendications s'expriment tout d'abord par l'intégration de traits d'oralité dans l'écrit, et de ce fait toute " irrégularité langagière » était marquée comme populaire tant que la langue littéraire revêtait la place de norme dans le roman (Rouayrenc, $2010: 235$ ). Au début du $20^{\text {ème }}$ siècle, les romancier-e-s vont se servir de la langue parlée comme «modèle formel » face à la langue écrite, faisant office de «levier argumentatif » dans le cadre d'une remise en cause de la littérature (Meizoz, 2007 : 39).

À l'instrumentalisation de la langue parlée s'ajoute la revendication d'une nouvelle esthétique littéraire qui va passer par un refus de ce que Meizoz (2007: 42) nomme le «cloisonnement des voix» dans la tradition littéraire jusqu'aux années 30, consistant à séparer le discours « familier » des personnages à celui littéraire du narrateur.

La figure du peuple est au cœur de ce renouveau, et les personnages du roman ont à ce propos un rôle central dans le langage utilisé, à travers la réflexion sur la présence des personnages populaires dans le genre littéraire : ceux-ci sont d'un double mouvement prétexte et vecteur de ce style (Rouayrenc, $2010: 235$ ).

Wolf (2007: 20-25) a théorisé cette idée de «fiction démocratique », dont la « diction démocratique » met en jeu la question de la présence du peuple dans le roman sur le plan linguistique, qui va passer par sa représentation dans la langue littéraire. Cette composante est une question à laquelle les écrivain-e-s apportent diverses réponses, se rassemblant sur l'idée d'une «complicité » entre la langue « qui signale la présence du peuple » et celle qui « signale l'activité de l'écrivain » (Wolf, $2007: 31$ ).

L'exemple des écrivain-e-s du « roman parlant» est révélateur de l'imbrication de ces différentes revendications : d'après Meizoz (2007: 58), leur utilisation du style oralisé s'unit dans une "médiation » entre " le renvoi à l'expression populaire comme référent à la fois éthique et stylistique », ce qui fait entrevoir un "fantasme de réconciliation » entre la langue du romancier et celle du lectorat, car, au-delà de ses revendications, l'écrivain-e reste un-e lettré-e et son rapport au peuple peut être questionnable.

En guise de conclusion, Sillam (1991: 49) qui a analysé la caractérisation des personnages du roman Bel-Ami de Maupassant identifie quatre grands axes sur lesquels peut s'appuyer l'écrivain-e pour son projet d'imitation de la langue parlée dans le roman :

1) La « situation de communication», englobant les échanges, leur organisation et la caractérisation des personnages, « axe principal de l'oralisation du discours »

2) La «distribution de la parole» entre les personnages, dont leurs « ressources verbales et non verbales » et l' « ajustement à la situation d'interlocution »

3) Les choix linguistiques variés réalisés sur la langue par les personnages, dont leurs « savoir-faire linguistiques » et « non linguistiques »

4) Les «modalités de déroulement des échanges» et les règles sous-tendant les « conduites sociales » des personnages par rapport au contexte du roman

\subsection{Variétés des imitations et niveaux d'oralité}

Si les buts des écrivain-e-s sont différents, la langue imitée l'est également, d'autant plus quand on sait que le style oralisé est un procédé réparti dans le temps et suivant donc les évolutions naturelles de la langue, même si nous allons voir que malgré cela les traits imités peuvent se révéler assez semblables à travers les différentes époques. 
Les phénomènes servant aux écrivain-e-s à connoter l'oralité dans leurs romans sont très différents les uns des autres et Rouayrenc (2010 : 235-236) les classe selon trois axes :

- Des atteintes à la Norme

- Des marques de registres

- Des faits propres à l'oral qui ne sont ni atteintes à la Norme ni marques de registre

On retrouve dans les atteintes à la norme le phénomène du «que " en tête de l'incise, les phénomènes de dislocations pour les marques de registres, et la marque de la suppression $\mathrm{du}$ « e » caduc pour le troisième axe (Rouayrenc, $2010: 235-236$ ).

L'autrice identifie dans un même temps trois niveaux d'oralité dans la littérature contemporaine en lien avec les trois axes ci-dessus (Rouayrenc, $2010: 248-250$ ) :

- L'oralité normée

- L'oralité courante

- L'oralité marquée

Nous avons déjà vu que les traits imités par les écrivain-e-s, loin de se limiter au lexique, pouvaient être trouvés dans différents domaines de la langue. Chaque domaine compte néanmoins des traits «majeurs» que Rouayrenc (2010:235-236) qualifie de « dénoteurs », tels que l'omission du «ne » de la négation, les marques de l'effacement du «e » caduc, ou encore le phénomène du « que » en tête de l'incise.

Ces « dénoteurs » constituent un « code du parler populaire » pour trois raisons : tout d'abord, on peut les retrouver dans divers romans; ensuite, comme dit plus haut, ils rassemblent en réalité des phénomènes linguistiques qui ne sont pas à mettre sous la même étiquette (Rouayrenc, 1996 : 32-34). Enfin, les dénoteurs fonctionnent en complémentarité au sein d' " un système dans lequel chacun est doté d'une valeur » (Rouayrenc, 1996 : 3334), selon la récurrence à laquelle les écrivain-e-s les emploient, le niveau d'entorse à la norme qu'ils représentent, et leur caractère optionnel.

À travers les conclusions provisoires de l'autrice ${ }^{\text {ii }}$, il semble qu'au premier abord leur évolution du début du $20^{\text {ème }}$ à la fin du siècle soit faible, mais on relève quelques modifications : à côté des phénomènes se maintenant, qui sont de manière générale des marques de registre, il semblerait que les marques de niveau soient moins utilisées, tel le phénomène du "que" servant de "cheville syntaxique après un adverbe ou un pronom interrogatif $[\ldots] »$ (Rouayrenc, $1996: 41-44$ ).

On peut se référer à l'étude de Favart (2010) pour l'analyse d'un corpus de romans de la fin du $20^{\text {ème }}$ au début du $21^{\text {ème }}$, dont la problématique est de déterminer si les phénomènes utilisés correspondent à un registre populaire, et, après confrontation avec un corpus de langue parlée, elle conclut que pas un seul phénomène imité « ne peut être considéré, dans la langue parlée authentique, comme socialement connoté. ».

Son explication est qu'il s'agit d'un phénomène de «décalage de registre linguistique », qui a pour conséquence d'augmenter la connotation d'un phénomène au point de rendre de l' «oral courant» en "oral socialement connoté » à l'écrit (Favart, 2010), ce que notent également Ravazzolo, Traverso, Jouin, et Vigner (2015: 180-181) sur les imitations phonétiques des romancier-e-s en décalage avec la réalité linguistique.

De plus, l'identification des registres se fait selon un échange entre l'écrivain-e et le lectorat; il représente un "artefact à deux niveaux » où l'écrivain-e va imiter la langue qu'il connait et qu'il identifie comme populaire, en lien avec «l'image que le lecteur a du sociolecte qui est représenté. » (Favart, 2010).

Les études se concentrent surtout sur les intentions des auteur-e-s, mais en réalité la représentation de la langue parlée fonctionne en miroir car les écrivain-e-s utilisent des phénomènes avec l'idée qu'une connotation sera partagée par les lecteurs-trices, ce qui pose la question de la « perception » de l'imitation (Rouayrenc, $2010: 235$ ).

Ainsi, quelles sont les représentations faisant qu'un trait va être considéré par le lectorat comme une marque de registre ou relevant de l'oralité, en un sens qu'est-ce qui «fait oral pour un lecteur ou une tradition interprétative donnée » (Mahrer, 2017:12)? 


\section{L'imitation littéraire de l'oral et la langue parlée}

\subsection{Préambule : contraintes et buts de l'analyse}

Suite à une présentation du style oralisé dans ses grandes lignes, nous nous concentrons désormais sur la convergence entre les imitations des écrivain-e-s et les études sur la langue parlée.

Avant cela, nous souhaitons préciser que le terme de "langue parlée » en lui-même est assez problématique dans une conception linguistique car il est difficile de conclure définitivement sur l'appartenance exclusive d'un phénomène à l'oral, et la question des caractéristiques générales de la langue parlée en elle-même se pose pour cette analyse.

Blanche-Benveniste (2007: 25) qui étudie six textes oraux de divers types et enregistrés dans diverses situations remarque par exemple qu'on ne relève pas un seul exemple de sujets disloqués, alors qu'on en retrouve pourtant un grand nombre dans les conversations, entre autres. Elle rappelle donc que certaines formes (dans son propos les dislocations) ne peuvent pas être considérées comme «caractéristique générale de tout ce qui est 'langue parlée' » mais plutôt comme une caractéristique des types de discours.

En effet, à part peut-être pour les traits phonétiques, même si la question de leur marquage orthographique à l'écrit amène de nouvelles problématiques, les traits imités ne relèvent pas tous définitivement du médium oral, et peuvent se retrouver dans certains types d'écrits, point sur lequel de récentes recherches se concentrent, comme McLaughlin (2011) sur les modalités d'utilisation de la dislocation dans les écrits journalistiques notamment.

L'analyse de deux extraits de romans (cf 2.3) aura pour but d'observer quels traits sont utilisés par les auteur-e-s, dans une mise en perspective de notre analyse avec les études déjà mentionnées. La question que nous nous posons donc à la suite de nombreux travaux est de savoir si nous pouvons trouver dans les imitations des écrivain-e-s des structures phonétiques, lexicales, syntaxiques présentes dans certains types de discours oraux.

\subsection{Jusqu'à quel point peut aller l'imitation ?}

Outre les difficultés liées à l'objet de définition de la langue parlée dans ses formes linguistiques, l'imitation de l'oral en littérature fait également face à d'autres contraintes.

Tout d'abord, le/la romancier-e n'est pas un-e linguiste, et de ce fait n'a peut-être pas les connaissances linguistiques nécessaires pour reproduire toutes les caractéristiques de la langue parlée (Durrer, $2005: 25$ ).

De plus, le canal utilisé représente une contrainte importante pour l'imitation, et enfin les écrivain-e-s ne veulent pas «choquer» leur lectorat et doivent aussi anticiper les obstacles à la lecture que leurs imitations vont représenter (Durrer, $2005: 25$ ).

Pour ces raisons, les imitations romanesques ne sauraient être un reflet exact de la langue parlée, et sont très souvent lacunaires sur certaines composantes fondamentales des conversations authentiques comme les régulateurs notamment (Durrer, $2005: 40$ ) ${ }^{\mathrm{iii}}$.

On ne peut donc pas imaginer y trouver toutes les caractéristiques de la langue parlée et à l'inverse les formes s'y trouvant n'appartiennent pas forcément à la langue parlée (Durrer, $1996: 68)$, ce qui ne signifie pas pour autant que les «instruments d'analyse » ne puissent pas être en partie communs (Durrer, $2005: 7$ ).

\subsection{Analyse de deux extraits de dialogues littéraires d'époques différentes}

Nous proposons une mise en miroir de deux extraits de dialogues de roman avec leur analyse linguistique succincte, dont un passage tiré d'Un homme, ça ne pleure pas (2015) de Faïza Guène et un extrait de Zazie dans le métro de Raymond Queneau ([1959] 2012). 
Nous avons choisi ces extraits pour pouvoir comparer deux époques, ainsi que des interactions ayant des caractéristiques différentes : l'extrait d'Un homme, ça ne pleure pas montre l'interaction entre deux jeunes adultes, alors que dans Zazie dans le métro les interlocuteurs sont une enfant et deux adultes.

- Dounia ?
- Oui ?
- Combien tu pèses ?
- Quest-ce que c'est que cette question?
- Je sais pas. T'es maigre, quand même.
- Ça se demande pas, ce genre de choses !
C'est comme l'âge !
- OK... J'voulais pas te vexer, désolé.
- T'as de ces idées, toi... »

Extrait tiré d'Un homme, ça ne pleure pas écrit par Faïza Guène aux éditions Le Livre de Poche, Paris, 2015, p.248.

- Et quand est-ce qu'elle va finir, cette
grève? demande Zazie en gonflant ses
mots de férocité.
- Je sais pas, moi, dit Gabriel, je fais pas
de politique.
- C'est pas de la politique, dit Charles,
c'est pour la croûte.
- Et vous, msieu, lui demande Zazie, vous
faites quelquefois la grève?
- Bin dame, faut bien, pour faire monter
le tarif.
Extrait tiré de Zazie dans le métro écrit par
Raymond Queneau aux éditions
Gallimard, Paris, [1959] 2012, p.14-15.

Concernant l'analyse linguistique de ces deux extraits, nous pouvons relever la présence de nombreux «dénoteurs»: la négation sans le «ne », la présence de dislocations, le marquage orthographique de plusieurs schwas, des élisions, et la suppression de « il » dans « faut bien ». On observe également le remplacement du « e » de « ben » par un « $\mathrm{i}$ », ainsi que plusieurs réalisations de la forme interrogative.

Les deux extraits utilisent donc au moins trois dénoteurs communs (la négation, les dislocations, les marques phonétiques) malgré le fait d'avoir été écrits à plus de cinquante ans d'intervalle, ce qui va dans le sens des remarques de Rouayrenc (1996 : 41-44), même

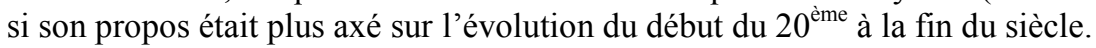

On note aussi dans les deux dialogues l'absence de régulateurs (à part peut-être pour le «bin »), ce qui corrobore les conclusions de Durrer (2005: 40) sur la sélection des écrivain-e-s concernant les caractéristiques de la langue parlée, et à ce propos, nous n'observons pas non plus de reformulations ou de faux-départs.

Enfin, on peut voir que le lexique utilisé n'est pas spécialement connoté de façon familière et ne présente aucune vulgarité, correspondant de même aux remarques de Durrer (2005 : 40) sur l'analyse d'un extrait de conversation authentique par rapport aux dialogues de romans imitant l'oral.

Concernant la comparaison des deux extraits, les répliques d'Un homme, ça ne pleure pas semblent plus courtes que celles de Zazie dans le métro, et ne sont pas non plus accompagnées de verbes introducteurs, ce qui semble donner un «effet» d'oralité plus important puisque tout le dialogue est au discours direct contrairement à Zazie dans le métro où les verbes introducteurs rompent en quelque sorte la vitalité des échanges.

En synthèse, malgré l'absence de certains éléments inhérents à la conversation authentique, on remarque que les imitations sont néanmoins riches de phénomènes qui peuvent être intéressants à exploiter didactiquement dans un travail sur les caractéristiques de certains types de discours oraux.

\section{Dimension didactique: comment exploiter les traits linguistiques imités en classe de langue}




\subsection{Rencontre littéraire avec l'objet de langue parlée}

Ce chapitre étudiera la manière d'utiliser les imitations de l'oral en littérature dans un objectif didactique et l'intérêt que ce matériel peut revêtir dans un contexte d'enseignement, plus particulièrement dans l'enseignement d'une langue étrangère en tant que sensibilisation à la langue parlée ; nous expliquerons également pourquoi nous avons élargi notre matériel en intégrant des extraits de corpus oraux dans la prochaine partie.

Un des articles les plus anciens sur le sujet est une étude de Calvi (1996) en didactique de l'espagnol; l'autrice y donne cinq arguments pour un usage du texte littéraire en classe (Calvi, 1996 : 110-112) que nous résumons :

- L'intemporalité du texte littéraire face à d'autres textes moins intemporels comme les documents journalistiques/radiophoniques, même s'il faut penser aux conséquences sur la langue selon son ancienneté

- La nature plurivoque de ce genre de texte, et le fait qu'il soit moins sensible au contexte de son élaboration que d'autres messages

- Leur fonction expressive transmise dans la langue

- Leur potentiel intérêt pour les apprenant-e-s selon leur sélection pertinente

- La grande diversité de la langue littéraire et le fait que les écrivain-e-s en donnent une importante variété de modèles qui ne s'arrête pas à une langue formelle

Dans cette optique, la lecture du texte littéraire pourrait selon les besoins de l'enseignant-e servir d'appui pour reconnaitre des phénomènes entendus dans des discours spontanés mais étant toujours en cours d'assimilation (Calvi, 1996 : 113).

En prenant pour exemple des extraits de romans espagnols de la seconde moitié du $20^{\text {ème }}$ siècle, elle propose un travail avec ces textes comme matériel pour découvrir des formes de vocabulaire familier, ou étudier les relations entre les personnages dans diverses situations de communication selon le langage employé (Calvi, 1996:114-117), et sa réflexion semble être une des premières, avec l'étude de Beltrán et Ripoll (1996).

Il semblerait que les travaux sur cet objet se trouvent particulièrement en didactique de l'espagnol : on peut citer plus récemment les travaux de Muñoz Medrano (2008), ou encore Camacho Guardado (2010).

En didactique de l'italien, Corbucci (2007) propose d'utiliser ce matériel pour aborder la variation sociolinguistique en classe avec trois extraits de textes d'époques et styles différents ; l'autrice montre avec ces textes la diversité d'usages de la langue dans l'écriture romanesque, dont une imitation des marques de l'italien neostandard avec la présence de régionalismes dans La bella di Lodi d'Alberto Arbasino, une langue plus contemporaine dans L'uomo che non ho sposato de Rosanna Campo, ou encore un langage d'un registre plus soutenu avec Alberto Moravia dans Il disprezzo.

En France, le travail le plus récent se trouve dans l'ouvrage de Weber (2013: 247-250), qui prend l'exemple d'un extrait du roman Ensemble c'est tout d'Anna Gavalda a dans une visée didactique en tant qu'exercice d' " observation » des phénomènes imités par l'écrivaine, avant une « sensibilisation par une action réfléchie ».

Comme il a été dit plus haut, l'utilisation didactique de ce matériel ne peut se faire sous couvert de représentation authentique de l'oral, étant un «système parallèle qui n'offre pas la présentation combinatoire propre au système de l'interaction orale. », même s'il semblerait qu'il « facilite certaines opérations de repérage [...] »(Weber, $2013: 49$ ).

On lui donne néanmoins une place dans les récents travaux sur la didactique de l'oral, comme l'ouvrage de Ravazzolo, Traverso, Jouin et Vigner (2015: 184), toujours en insistant sur le fait qu'il ne saurait être « une représentation mimétique de la conversation naturelle ».

La question se pose également de savoir à quel niveau introduire ce matériel particulier d'oral représenté en classe: pour Calvi (1996) et Corbucci (2007), il serait plutôt à présenter aux niveaux avancés, et seule Weber (2013 : 249-250) semble suggérer une idée de progression, selon le niveau des élèves mais aussi selon les publics concernés. 


\subsection{Entre oral et écrit}

Ce matériel par sa forme vient questionner les modalités d'écrit et d'oral, et en particulier le lien entre ces deux codes, qui en didactique semble osciller entre articulation et opposition.

Les réflexions sur « «l'oral dans l'écrit»» et « «l'écrit dans l'oral» " ne cessent en effet de " captiver les chercheurs », même s'il faut aussi prendre en compte la " large zone d'intersection entre les deux modalités », qui « rend toute entreprise de délimitation stricte de frontières caduque » (Gagnon, De Pietro \& Fisher, 2017 : 24).

À ce titre, les auteurs proposent dans la préface de leur ouvrage que le fait d'observer «diverses sortes d'oral, des intersections entre langue orale et écrite, des diverses réalisations langagières d'un même genre » serait à même de "favoriser la réflexivité de l'élève et l'amener à avoir un rapport plus conscient au langage. » (Gagnon, De Pietro et Fisher, 2017 : 30). Cette perspective est aussi la nôtre et celle que nous poursuivons grâce à l'utilisation de notre matériel.

En effet, les écrivain-e-s ont pensé les liens entre l'écrit et l'oral, et leurs romans sont des documents de choix pour aborder cette problématique, en particulier les auteurs du $20^{\text {ème }}$ siècle selon Baptiste (2015:214), qui, dans un renouveau de l'écriture romanesque, ont également «modifié notre perception de la langue même et de ses codes. ».

De ce fait, leurs écrits sont : « un terrain privilégié de réflexion sur la langue pour un apprenant. » (Baptiste, 2015 : 214).

Enfin, cette utilisation du texte littéraire en classe en tant que matière à réflexion pourrait aussi conduire les apprenant-e-s à étudier les modalités d'usage de la langue, dont le fait de «relativiser l'importance encore souvent accordée à la norme, ou du moins à l'envisager comme un facteur qui peut être limitant [...]» (Baptiste, $2015: 209$ ).

\subsection{Perspective intercompréhensive de la présentation du matériel}

La visée originale de notre recherche est d'utiliser le matériel dans une perspective intercompréhensive, en proposant de manière simultanée un travail sur un extrait littéraire en langue cible, un extrait en langue première et un extrait dans une troisième langue romane.

Cette approche fait non seulement écho aux réflexions sur la didactique des langues voisines (Dabène, 1975), mais plus encore à celles de l'intercompréhension en didactique (Escudé \& Janin, 2010), approche dans laquelle nous nous inscrivons.

Des dispositifs binationaux de double diplôme à l'instar du parcours EsaBac en France et en Italie intègrent en un sens cette dimension dans leur programme de littérature, en élaborant des curricula conjoints d' " «itinéraires littéraires » » avec des comparaisons entre des extraits de mêmes époques et thématiques comme « «le Réalisme et le Naturalisme en France ; il Verismo en Italie » »(Weeger, 2015 : 125-127).

Même si notre approche n'est pas celle de la littérature comparée car les extraits sont plus prétextes à un travail d'intercompréhension et un travail sur la langue que sur l'écrivain-e et son courant littéraire, nous retrouvons effectivement dans ces orientations la visée de mettre en perspective des extraits de textes littéraires en différentes langues pour étudier les liens les unissant.

\section{Présentation de l'utilisation du matériel en classe}

\subsection{Objectifs et organisation du matériel avec l'utilisation de corpus oraux}

Au moment de l'écriture de cet article, le matériel créé est celui utilisé dans le cadre de l'expérimentation de notre premier terrain qui se déroule dans les cours d'italien langue 
étrangère au sein d'un lycée en France pour un public d'élèves de la seconde à la Terminale.

L'organisation du matériel créé se conçoit comme des séances individuelles réalisables en une heure, prenant pour départ un point de langue touché par des phénomènes de variation à l'oral (la négation, les dislocations, les phénomènes d'apocopes), exemplifiés grâce à la présentation de trois extraits de dialogues littéraires et trois extraits de corpus oral concernant le même fait linguistique.

Nous avons choisi d'utiliser principalement le corpus oral multilingue C-Oral-Rom pour la partie d'écoute de nos activités, même si nous empruntons également ponctuellement des extraits dans des corpus oraux unilingues selon nos besoins.

Le corpus C-Oral-Rom est composé d'extraits en quatre langues romanes (le français, l'italien, l'espagnol et le portugais) coordonné par des équipes des universités d'AixMarseille, de Florence, de Madrid, et de Lisbonne (Deulofeu \& Caddéo, 2009 : 153).

Il a été créé pour répondre à un besoin de "données linguistiques en contextes «naturels» diversifiés », aussi bien pour la recherche en linguistique que pour les « enseignants qui cherchent à étendre à l'oral l'utilisation de documents authentiques dans leur démarche pédagogique. » (Deulofeu \& Caddéo, 2009 : 153-155).

Notre souhait d'utiliser en complément des ressources écrites des extraits de corpus oraux correspond d'une part à un besoin lié à notre recherche, car le fait de présenter seulement des extraits écrits nous semblait peu pertinent et nous souhaitions apporter un exemple oral des traits sélectionnés dans un exercice d'écoute.

D'autre part, cela correspond aux premières réflexions sur l'intercompréhension orale montrant l'intérêt du recours au matériel écrit et l'articulation entre les deux codes dans une tâche de compréhension orale entre langues voisines (Jamet, 2005 : 66-67).

Enfin, cela fait écho aux préoccupations actuelles de la recherche en didactique des langues sur l'utilisation de corpus oraux pour présenter des discours plus authentiques aux apprenant-e-s, même si l'écoute de discours spontanés est une composante à laquelle LebrePeytard (1990:10) appelait déjà il y a trente ans pour permettre :

- Une «réflexion sur la cohérence du discours entendu et sur les relations entre productions langagières et paramètres socio-situationnels "

- Une « comparaison entre ce discours et d'autres productions orales »

- Une « sensibilisation aux registres de langage »

- Une « remise en question du concept de norme au profit de celui de normes langagières »

Actuellement, la question se pose en effet d'utiliser les corpus oraux pour sensibiliser les apprenant-e-s à des usages de la langue orale que l'on trouve difficilement dans les ressources pour l'enseignement, comme le très récent projet de corpus pour l'enseignement du FLE, FLORALE (Surcouf \& Ausoni, 2018).

Dans cette optique, l'ouvrage coordonné par Detey, Durand, Laks et Lyche (2010) Les variétés du français parlé dans l'espace francophone : ressources pour l'enseignement est un exemple de travail réunissant ces problématiques en proposant un ensemble d'extraits oraux authentiques accompagnés de leur analyse linguistique et de pistes d'exploitation didactique en classe.

\subsection{Déroulement d'une séance en classe}

Les activités que nous proposons en classe sont organisées en deux temps : la première partie est consacrée à l'exercice de compréhension écrite des trois extraits des dialogues de romans dans une présentation des documents s'organisant avec :

1) Une introduction au texte en langue cible pour présenter le roman, son auteur/autrice et son contexte historique/spatial 
2) L'extrait en langue cible, accompagné d'un tableau récapitulant la situation de communication pendant l'échange (les interlocuteurs présents, les lieux dans lesquels ils se trouvent, l'objet de la discussion)

3) Des questions de compréhension et de repérage pour guider la lecture du texte et amener l'attention des apprenant-e-s sur le phénomène ciblé par l'activité

4) Les deux autres extraits (un extrait en français et un extrait dans une troisième langue romane, l'espagnol ou le portugais) accompagnés d'une question de compréhension/repérage en lien avec le phénomène présent dans l'extrait italien

La deuxième partie de l'activité est consacrée à l'écoute des trois extraits oraux, où un espace pour chaque document est attribué dans la fiche de l'activité de manière à pouvoir prendre des notes pendant l'écoute et présenter une transcription.

Nous avons en effet choisi de proposer une transcription pour accompagner l'extrait dans la troisième langue romane inconnue et apporter une aide visuelle à l'apprenant-e pendant l'écoute car le but de la tâche demandée n'est pas celle d'une activité d'intercompréhension orale au sens strict du terme mais un exercice de repérage.

Pour la langue cible et la langue de scolarisation en revanche, nous avons fait le choix de ne pas proposer de transcription, une modalité dont l'incidence sera à analyser dans nos résultats, et éventuellement à modifier pour le second terrain.

La séance se conduit sur un créneau d'une heure mis à disposition par l'enseignante d'italien de la classe qui est présente à nos côtés pendant les activités ; les apprenant-e-s sont répartis en groupe de quatre à cinq élèves, et sont enregistrés à l'aide de dictaphones pour recueillir les interactions servant à l'analyse des données.

Si les élèves sont en autonomie pendant la réalisation des activités, l'enseignante et moi-même sommes disponibles pour des questions éventuelles et nous contrôlons également la réalisation de l'activité en passant régulièrement dans les groupes.

Le déroulement des activités est pensé pour aborder la partie écrite dans un premier temps puis la partie orale en dernier, même si nous avons pu voir que la gestion du temps était souvent délicate, et malgré nos efforts pour présenter des extraits courts, la partie écrite débordait souvent sur le temps imparti et empiétait sur la partie d'écoute.

\subsection{Perspectives pour l'analyse des résultats de l'utilisation en classe}

Les perspectives d'analyse de l'ensemble des données s'articulent avec les enregistrements des séances de classe constituant le cœur de notre analyse ; en complément, nous mettons également en place deux questionnaires pour les apprenant-e-s, dont le premier se tient au début de la recherche et le second à sa conclusion, et nous réalisons un entretien semi-guidé des enseignant-e-s des classes dans lesquelles nous réalisons les expérimentations.

L'analyse croisée de ces données visera à observer dans un premier temps l'incidence de l'utilisation de notre matériel sur la compréhension des usages oraux de la langue cible.

Comme nous avons essayé de le démontrer avec les études sur le sujet (Weber, 2013 ; Calvi, 1996), nous pensons que l'imitation littéraire des traits de l'oral peut être pertinente pour nos objectifs d'apprentissage : nous avons vu que l'inventivité des auteurs et des autrices est en effet riche à exploiter didactiquement en lien avec des problématiques sur l'oral et l'écrit.

Si les traits imités par les écrivain-e-s ne peuvent être qualifiés d'oral authentique, ceux-ci peuvent néanmoins amener les apprenant-e-s à réfléchir sur cet objet particulier de l'oral dans l'écrit pour ensuite aborder la question des usages de la langue parlée.

En particulier, nous pensons que la présentation des traits de la langue orale à l'écrit est un déclencheur intéressant pour les apprenant-e-s se retrouvant confrontés à la présence d'un code hors de son canal d'utilisation avec toutes les particularités que cela implique. 
Notre hypothèse est que ces activités vont également pouvoir faire s'interroger les élèves sur leur propre utilisation de la langue en lien avec les traits imités; en tant que matériel réflexif, ces imitations vont être source de jugement pour les élèves, qui vont pouvoir confronter leurs représentations de la langue parlée à celles des écrivain-e-s.

Le but étant de les amener à discuter de l'usage de ces traits, d'une part selon la façon dont ils sont présentés dans la représentation qu'en font les auteur-e-s, et d'autre part dans leur propre utilisation quotidienne, selon différents facteurs liés aux contextes d'utilisation de la langue que nous chercherons à faire expliciter aux élèves.

Notre hypothèse est qu'une telle modalité puisse pousser les apprenant-e-s non seulement à réfléchir et peut-être modifier leurs propres représentations de l'usage de l'oral en langue première, mais surtout les amener à créer des connaissances et des représentations sur l'usage en langue cible, de manière à pouvoir reconnaitre les traits utilisés à l'oral et être en mesure de comprendre leur utilisation.

Notre travail vise en effet un résultat de sensibilisation aux traits de la langue orale, et s'affranchit donc de la question de leur production.

La particularité de notre étude sera également à mesurer dans l'apport de la troisième langue dans une perspective intercompréhensive, en étudiant ce que l'extension d'un modèle par la confrontation à une diversité de réalisations peut avoir comme effet en termes de compréhension et de fixation des usages étudiés.

\section{Conclusion}

A l'issue de cet article où nous avons présenté une partie du travail d'expérimentation de notre thèse, les perspectives d'exploitations futures du matériel créé restent importantes.

D'après nos premières observations, nous retenons pour le moment dans la réalisation des activités en classe l'importance de faire s'exprimer les apprenant-e-s sur les documents mis à leur disposition.

En effet, nos premières impressions sont que le potentiel de réflexion du matériel littéraire semble conséquent, tant au niveau de la langue utilisée par les auteur-e-s que sur les propres représentations des apprenant-e-s par rapport à leur utilisation de la langue.

Réfléchir à la manière dont est présenté un phénomène par l'écrivain-e et comment celui-ci peut être utilisé dans son propre répertoire langagier est un exercice particulier pour l'apprenant qui est confronté à son propre usage grâce à un matériel déclencheur, qui a pour but final de créer de nouvelles modalités d'usage en langue cible.

L'intérêt d'utiliser ce matériel se conjugue également avec plusieurs difficultés liées à celui-ci, comme l'imitation que font les écrivain-e-s de la langue qui peut tomber dans un stéréotype linguistique (Favart, 2010) ; néanmoins, nous pensons que les apprenant-e-s peuvent réfléchir à cette composante qui est soutenue par la présentation d'un même fait en plusieurs langues.

Le défi de notre étude est également de mettre en perspective la présentation d'un même fait en plusieurs langues dans la sélection des extraits, et de mettre à profit cette modalité de travail plurilingue dans l'exercice de compréhension en classe.

\section{Références bibliographiques}

Baptiste, A, Woerly, D \& Lumbroso, O. (2015). Chapitre 5. Le rôle de la littérature dans les apprentissages langagiers : de l'écriture créative à la conscience de la langue. Dans Godard, A. (dir.), La littérature dans l'enseignement du FLE, p.169-219. Paris : Didier.

Beltrán, M. J., \& Ripoll, B. (1996). Ficcionalización de oralidad : propuestas para la enseñanza del español hablado en las clases de E/LE. Dans Grande Alija, F. J., Le Men, Y., Rueda, M. \& Prado Ibán, E. (coord.), Actas del VI Congreso Internacional de ASELE: Actuales tendencias en la enseñanza del español como lengua extranjera I, León 5-7 octobre, p. 73-78. 
Blanche-Benveniste, C. (2007). Le français parlé au $21^{\mathrm{e}}$ siècle : Réflexions sur les méthodes de description: système et variations. Dans M. Abecassis, L. Ayosso \& E. Vialleton (éds.), Le français parlé au XXIe siècle: normes et variations géographiques et sociales, p.17-39. Paris : L'Harmattan.

Calaresu, E. (2005). Quando lo scritto si finge parlato. La pressione del parlato sullo scritto e i generi scritti più esposti : il caso della narrativa. Dans Hölker, K. \& Maßß, C. (a cura di), Aspetti dell'italiano parlato, p. 81-115. Münster : Lit Verlag.

Calvi, M. V. (1996). Dialogo reale e dialogo letterario : prospettive didattiche. Dans Atti del convegno : Lo spagnolo d'oggi : forme della comunicazione, Rome, 15-16 mars, p. 107-117. Roma : Bulzoni.

Camacho Guardado, A. L. (2010). El español coloquial en contextos académicos : estudio a través del texto literario Las mil noches de Hortensia Romero de F. Quiñones. Dans Vera Luján, A. \& Martínez Martínez (éds.), El español en contextos específicos : enseñanza e investigación, p. 1055-1070. Fundación Comillas.

Corbucci, G. (2007). Fenomeni di variazione sociolinguistica nell'insegnamento dell'italiano a stranieri. Studi di glottodidattica, 1(2), 93-115.

Dabène, L. (1975). L'enseignement de l'espagnol aux francophones (Pour une didactique des langues « voisines »). Langages, 39, 51-64.

Deulofeu, J., \& Caddéo, S. (2009). Nouveaux défis et nouveaux outils pour l'étude des langues romanes : l'enseignement simultané (EUROM4) et les corpus oraux parallèles (C-ORAL-ROM). Studia Universitatis Babes-Bolyai, Philologia, 54(3), 152-164.

Detey, S., Durand, J., Laks, B. \& Lyche, C. (2010). Les variétés du français parlé dans l'espace francophone. Ressources pour l'enseignement. Paris : Ophrys.

Duarte, I. M. (2012). Comment la fiction traduit-elle l'oralité en portugais ? Dans Araújo Carreira, M. H. (org.), Les rapports entre l'oral et l'écrit dans les langues romanes, Série Travaux et documents, p. 447-462. Saint-Denis : Université Paris 8.

Durrer, S. (1996). Style oralisé et fuite du sens. Réflexions autour de "La grande peur dans la montagne" de Ramuz. Versants, 30, 63-84.

Durrer, S. (2005). Le dialogue dans le roman. Paris : Armand Colin.

Escudé, P. \& Janin, P. (2010). Le point sur l'intercompréhension, clé du plurilinguisme. Paris : CLE International.

Favart, F. (2010). Le stéréotype de registre de langue populaire dans le roman du second XXe siècle (1966-2006). Textes et contextes, 5. URL : http://preo.ubourgogne.fr/textesetcontextes/index.php?id=255

Gagnon, R., De Pietro, J-F., Fisher, C. (2017). L'oral aujourd'hui : perspectives didactiques [Introduction]. Dans De Pietro, J-F., Fisher, C. \& Gagnon, R. (dirs.), L'oral aujourd'hui : perspectives didactiques, p. 11-40. Namur : Presses universitaires de Namur.

Havard, A-M, \& Weeger, M. (2015). Chapitre 3. L'enseignement de la littérature de langue français à l'étranger: lieux, dispositifs et tendances. Dans Godard, A. (dir.). La littérature dans l'enseignement du FLE, p. 91-129. Paris : Didier.

Jamet, M-C. (2005). L'intercompréhension orale entre langues voisines : réelle possibilité ou illusion ? Synergies Italie, 2, 61-68.

Kim, M. (2014). Réflexions sur le dialogue littéraire dans les manuels de FLE pour l'apprentissage langagier et littéraire en classe universitaire. Colloque international des Etudiants chercheurs en Didactique des langues et en Linguistique, Lidilem, Juin 2014, Grenoble.

Lebre-Peytard, M. (1990). Situations d'oral : documents authentiques : analyse et utilisation. Paris : CLE International. 
López Serena, A. (2007). Oralidad y escrituralidad en la recreación literaria del español coloquial. Madrid : Gredos.

Luzzati, F., \& Luzzati, D. (1987). Oral et familier : Le style oralisé. L'Information grammaticale, $34(1), 15-21$.

Mahrer, R. (2017). Phonographie : la représentation écrite de l'oral en français. Berlin : De Gruyter.

Manno, G. (1996). Cassola neorealista, ovvero tra oggettivismo e mimetismo : un'analisi linguistica della « Ragazza di Bube ». Versants, 30, 137-161.

Muñoz Medrano, M. C. (2008). Aproximación a la sintaxis coloquial a través del diálogo literario : aplicación en la clase de E/LE. Dans Actas del II Congreso Internacional de Lengua, Literatura y Cultura de E/LE : Teoría y práctica docente, Valencia, 15-17 mai, p. 215-228.

McLaughlin, M. (2011). When written is spoken : dislocation and the oral code. Journal of French Language Studies, 21(2), 209-229.

Meizoz, J. (2001). L'âge du roman parlant (1919-1939) : écrivains, critiques, linguistes et pédagogues en débat. Genève : Droz.

Meizoz, J. (2007). Techniques et idéologies à l'âge du "roman parlant", 1919-1939. Dans Petitjean, A., \& Privat, J. M. (dirs.), Les voix du peuple et leur fiction, 39-60. Metz : Editions de l'Université de Metz.

Ravazzolo, E., Traverso, V., Jouin, E., \& Vigner, G. (2015). Interactions, dialogues, conversations : l'oral en français langue étrangère. Paris: Hachette.

Rouayrenc, C. (1996). Le parlé dans le roman : variations autour d'un code. Versants, 30, 31-44.

Rouayrenc, C. (2010). Le français oral 2 - L'organisation et la réalisation de l'énoncé oral. Paris : Belin.

Sillam, M. (1991). La variation dans les dialogues de Bel-Ami. Langue française, 89, 35-51.

Surcouf, C., \& Ausoni, A. (2018). Création d'un corpus de français parlé à des fins pédagogiques en FLE : la genèse du projet FLORALE. EDL, 31, 71-91.

Vigneau-Rouayrenc, C. (1991). L'oral dans l'écrit : histoire (s) d'E. Langue française, 89, 20-34.

Weber, C. (2013). Pour une didactique de l'oralité. Enseigner le français tel qu'il est parlé. Paris : Didier.

Wolf, N. (2007). Fiction et diction démocratiques. Dans Petitjean, A., \& Privat, J. M. (dirs), Les voix du peuple et leurs fictions, 19-37. Metz : Editions de l’Université de Metz.

\footnotetext{
${ }^{\text {i } V o i r ~ l ' a n a l y s e ~ d e s ~ p r e m i e r s ~ c h a p i t r e s ~ d e ~ s o n ~ r o m a n ~ L a ~ r a g a z z a ~ d i ~ B u b e ~ p a r ~ M a n n o ~(1996) . ~}$

${ }^{\text {ii }}$ Dans l'attente de l'étude d'un corpus plus important (l'autrice précise).

iii Voir aussi Ravazzolo, Traverso, Jouin, Vigner (2015 : 180-184).

${ }^{\text {iv }}$ Voir aussi Kim (2014) sur l'utilisation des dialogues littéraires en FLE.
} 